\title{
Die illegitime Zeit: Ästhetik und politische Theologie
}

\begin{abstract}
For a long time in morphological thought and debate there was gap regarding political morphology. The question of katechon, of the withholding power, developed from St. Paul's Second Letter to Thessalonians (2:6, in particular), gave rise to a long string of interpretations that continues to this day. The idea of katechon is connected to the legitimacy and stability of political power. It is very important to discover the possible connections between the political and the aesthetical forms in their capacity to offer a solution to an unconnected multiplicity (despite their different contents).
\end{abstract}

\section{Katechon und Legitimität unserer Zeit}

Die Metapher des Katechon bzw. der „aufhaltenden Macht“ besitzt eine erstaunliche Aktualität in einer Welt wie der unseren. Von Ressentiment und Vergangenheitsnostalgie, von Nationalismen und Lokalpatriotismen beherrscht, hat diese Welt die traditionellen Legitimitätsformen aus dem Blick verloren. Allerdings ist das Thema der Legitimität der Neuzeit - um es mit dem Titel des berühmten Buches von Hans Blumenberg zu sagen - durchaus nicht neu, sondern hat weit zurückreichende Wurzeln (Blumenberg 1988). Unsere Gegenwart erlebt keine unerwartete Krise. Die entropischen und zentrifugalen Kräfte waren bereits ein typisches Merkmal der frühen Neuzeit und der reifen Moderne - um einen Bogen zu spannen, der bei den Poetiken der deutschen Frühromantik ansetzt und in dem großen Essay von Ernst Jünger, Die totale Mobilmachung, gipfelt. Wir haben es mit einer großen Angst der Moderne vor einer Zeit der Entropie zu tun, worunter ich hier im weiten Sinn eine Tendenz zum Energieverlust meine. Es ist ein langer Weg, der von größter Bedeutung ist, um zu erfassen, was derzeit geschieht. Man darf sich diesbezüglich nicht durch die enorme Beschleunigung täuschen lassen, die unsere Zeit erfasst hat. In Wirklichkeit ist das heutige Panorama nicht neu, sondern von langer Hand vorbereitet.

Vor diesem Hintergrund ist das wachsende Interesse der italienischen Philosophie für die politische Theologie einzuordnen. Bekanntlich rückte das Thema in den fünfziger Jahren mit der Publikation von Carl Schmitts Der Nomos der Erde in den Mittelpunkt der philosophischen Reflexion. Angefangen bei Massimo Cacciari über Roberto Esposito bis zu Giorgio Agamben hat sich die Reflexion über

Ә OpenAccess. ( 2021 Federico Vercellone, publiziert von De Gruyter. (cc))BY-NC-ND Dieses Werk ist lizenziert unter einer Creative Commons Namensnennung - Nicht kommerziell - Keine Bearbeitung 4.0 International Lizenz. https://doi.org/10.1515/9783110674194-015 
das Katechon in einem Rahmen entfaltet, in dem die Auslegung der neutestamentlichen Frage dem Verständnis und Selbstverständnis unserer Zeit den Weg bahnt. Es handelt sich um eine rein politisch-philosophische Frage, die nicht zufällig im Rahmen der Theologie und Religionsgeschichte wenig Interesse geweckt hat (z. B. fehlt im Index patristicus das entsprechende Stichwort). Wie ich in meinem Beitrag zeigen möchte, ist inzwischen auch die ästhetische Seite der Frage, die mit der Idee des Katechon als einer Form, die festhält und bewahrt, zusammenhängt, offen zutage getreten. Genau auf der ästhetischen Ebene wurde auch die grundlegende Unterscheidung zwischen Legitimität und Legalität getroffen, die aus dem Streitgespräch zwischen Schmitt und Blumenberg hervorging (Schmitt 2007).

\section{Das Katechon zwischen Antike und Moderne}

Die Frage des Katechon hat wichtige Vorläufer in der antiken Philosophie und im Alten Testament, doch ihre volle Reichweite entfaltet sich dann bekanntlich im Zweiten Brief des Paulus an die Thessalonicher, wo behauptet wird, dass das Kommen des Antichrist bevorstehe, aber von einem Katechon, einer ,aufhaltenden Macht“, aufgehalten werde. Diese spielt eine zugleich positive und negative Rolle. Einerseits wendet sie das Kommen des Antichrist und folglich ein radikales Übel ab; andererseits verhindert sie auch die Parusie Christi, der den Widersacher zerstören müsste, vorausgesetzt, dieser enthüllt sich. Paulus bezieht sich auf alttestamentliche Quellen, die das negative Gesicht des Katechon unterstreichen, also das, was das Kommen des Messias verhindert. (Es sei daran erinnert, dass die religiösen Parteien in Israel noch heute den weltlichen vorwerfen, dass sie das Kommen des Messias aufhalten.) Die Frage entwickelt sich dann in einem sehr ausgedehnten Zeitraum weiter, beginnend bei Hieronymus über Chrysostomus und Tertullian bis zu Konstantin I. und Eusebius von Caesarea und zur Reformation, zu Luther und Calvin. Sie durchzieht dann das russische Denken, von Rozanov bis Šestov, und findet schließlich ihren Höhepunkt in der großen Auseinandersetzung zwischen Carl Schmitt und Jacob Taubes, über welcher der Schatten von Walter Benjamin steht. Doch was steht bei dieser tausendjährigen Geschichte auf dem Spiel? Nichts anderes als die Legitimationsform der politischen Macht. Auf der anderen Seite geht es jedoch - und hier liegt das eigentliche Problem - um die Frage der illegitimen und ideologischen Macht und die Ideologie der Macht im Sinne von trügerischen und verlogenen Formen.

Wie gesagt, nimmt die Frage des Katechon in dem Moment eine seltsame Wendung, in dem die aufhaltende Macht eine Grenze für die Parusie Christi darstellt. Von Irenäus bis zu Tyconius und Hieronymus erscheint der Katechon, 
gleich ob er direkt thematisiert wird oder nicht, als eine Verhinderung der Parusie Christi. Er ist somit negativ konnotiert. Bei Hieronymus wird er als das Reich verstanden, das die Wiederkehr Christi verhindert, bei Irenäus und Tyconius als der dunkle Teil der Kirche (Tyconius, Liber regularum, 383). Augustinus war derjenige, der Rom, im Grunde auf den Spuren von Tertullian, durch die Trennung zwischen Reich Gottes und Reich der Welt, wenigstens in Bezug auf die rein politische Sphäre, eine zentrale Bedeutung zuschrieb. Der Konflikt der Reiche, der sich hinter der Frage des Katechon verbirgt, wurde damit zum Schweigen gebracht. In Contra Apologeticos betrachtet Tertullian das Reich als eine wahre Bremse, als ein Katechon, das die endgültige Auflösung bzw. das Weltenende aufhält, welches er nicht - aus einer messianischen Perspektive - als positives, sondern als negatives Ereignis bewertet. In seinen Augen überdauert das Reich die römische Zivilisation und ihre Laster bei weitem (Tertullian, Apologeticus adversos gentes pro Christianis).

In der ersten Phase ihrer Geschichte schließt die Frage des Katechon also, mehr oder weniger verhüllt, die des Konflikts der Reiche ein. Es geht um die bedeutsame Frage nach dem Ort des Reichs Gottes, das nicht auf der Vertikalen der Transzendenz, sondern auf der Horizontalen einer historisch-messianischen Bestimmung angesiedelt wird, was potenziell konfliktreiche Folgen für das eine wie das andere Reich mit sich bringt. Auf diese Phase folgt eine Art Konstruktion der (und Zwang zur) Transzendenz. Mit anderen Worten lässt sich die Hypothese aufstellen, dass die Transzendenz, die als Vertikalisierung des Glaubens definiert werden kann, zum Teil in der Frustration über die ausgebliebene Wiederkehr Christi ihren Ursprung hat. Mit diesem Schritt, der sich in Augustinus anbahnt, entsteht, wie gesagt, eine Art Verschiebung der Ebenen, wodurch die Schlüssel des Himmelsreichs und die des irdischen Reichs und seiner Regierung unterschiedlichen Obrigkeiten anvertraut werden. Die Transzendenz und ihre Konstruktion ermöglichen so eine Ordnung, die ursprünglich entgegengesetzte Instanzen ausgleicht und integriert. Mit diesem Übergang kehrt sich das Vorzeichen des Katechon um: Es erhält nun eine ausschließlich positive Bedeutung und verliert die negative Seite, die ihm zunächst eigen war. Seinen idealen Höhepunkt findet dieser Prozess viele Jahrhunderte später in Dantes De Monarchia, wo der Ausgleich zwischen beiden Momenten auf eine vollkommene Ordnung vorausweist:

\footnotetext{
Alle obigen Gründe bestätigt eine merkwürdige Erfahrung, nämlich jener Zustand der Menschen, welchen der Sohn Gottes, als er zum Heil des Menschen den Menschen anziehen wollte, entweder erwartete, oder, weil es sein Wille war, selbst anordnete. Denn wenn wir vom Fall der ersten Menschen, als dem Anfang unsers ganzen Irrweges, die Anordnungen der Menschen und die Zeiten durchblicken, so werden wir finden, daß nur unter dem göttlichen Augustus als Monarchen die Welt in einer vollkommenen Monarchie ruhig gewesen
} 
sei. Und daß das Menschengeschlecht damals glücklich war in der Ruhe des allgemeinen Friedens, das haben alle Geschichtschreiber, alle erlauchten Dichter, ja auch der Aufzeichner der Langmuth Christi für werth gehalten zu bezeugen. Endlich nannte auch Paulus jenen glücklichsten Zustand die Erfüllung der Zeit. Und in der That verdienten Zeit und alles Zeitliche den Ausdruck der Fülle, weil kein Geheimniß unsers Glückes eines Dieners ermangelte. Wie es aber mit dem Erdkreise bestellt gewesen sei, seitdem jenes unzerreißbare Gewand durch die Kralle der Begierde uranfänglich einen Riß erlitten habe, können wir theils lesen, theils, wollte Gott, nicht erblicken. (Dante Alighieri ca. 1316/1845, 25-26). ${ }^{1}$

Von nun an löst die Vorstellung von einem Verlust oder Untergang des Katechon eine abgrundtiefe Nostalgie und Melancholie aus, die nicht die ausgebliebene Apokalypse betrifft, sondern die Furcht, sie könne sich verwirklichen. Es ist die Furcht vor einer nicht wiedergutzumachenden Katastrophe, die es um jeden Preis zu vermeiden gilt. Dies ist im Grunde der Schritt, der uns zum letzten Kapitel der Geschichte führt, in dessen Mittelpunkt das Dreieck Schmitt - Taubes - Benjamin steht. Hier kreist die Auseinandersetzung um zwei einander gegenüberstehende Positionen: zum einen die schmittsche Apologie des Katechon als die aufhaltende Macht, die den letzten Schutzwall gegen das herannahende nihilistische Chaos darstellt; zum anderen die erneute Notwendigkeit einer messianischen Perspektive, die über die Ruinen der Geschichte hinausweist, deren barbarisches Gesicht Walter Benjamin und Jacob Taubes Recht gibt.

\section{Wege des Katechon}

Die Auseinandersetzung mit der Geschichte des Katechon in einer auf die Gegenwart gerichteten Perspektive bedeutet kurz gesagt, von der rein theologischen zur politisch-philosophischen Sphäre überzugehen, das heißt in die historische Sphäre der weltlichen Macht einzutauchen. Dieser Sphäre gehört das Katechon in

1 „A tutte le argomentazioni che precedono dà conferma un fatto memorabile: cioè la situazione dell'umanità che il figlio di Dio o attese, o quando volle, egli stesso predispose prima di farsi uomo per la salvezza degli uomini. Se infatti ripercorriamo la storia dell'umanità a partire dalla caduta dei nostri primi parenti, che fu la falsa via, origine di ogni nostro traviamento, troveremo che solo sotto la perfetta Monarchia del divo Augusto il mondo fu tutto in pace. E che allora il genere umano sia stato felice nella tranquillità di una pace universale, lo ammettono tutti gli storici; lo attestano poeti illustri e si è degnato di attestarlo anche l'interprete della mansuetudine di Cristo; ed infine Paolo chiamò questo stato felicissimo „pienezza dei tempi“. E veramente pieni furono i tempi e le cose temporali tutte, perché nessuna istituzione per la nostra felicità mancò allora di chi la rendesse efficiente. Ma come sia diventato poi il mondo da quando questa tunica inconsutile dovette per la prima volta subire la lacerazione da parte dell'artiglio della cupidigia, lo possiamo leggere, e vorremmo, ahimè, non vederlo“. 
den jüngeren Interpretationen an, die es mal im Reich, mal in der Katholischen Kirche verkörpert sehen. Die antike Geschichte des Katechon konfrontiert uns, wie gesagt, mit einer Verwandlung seiner inneren Struktur, die seine Bedeutungsordnung von Grund auf verändert. Das Katechon, das in der paulinischen Interpretation zunächst eine rein negative Bedeutung besaß, erhielt in der lateinischen Patristik über Tertullian und Chrysostomus eine positive Konnotation. Für die abendländischen Legitimationsformen der Macht ist die Frage von kaum zu überschätzender Bedeutung (Cacciari 2018). Die Reichsform emanzipiert sich nach und nach vom Römertum, um sich als unzeitliche Form der politischen Macht darzustellen, und die Frage der legitimen Herrschaft ist vollkommen mit der Idee einer monokratischen Herrschaft verknüpft, die nur einen Herrscher, nur einen legitimen Archon zulässt. Alles andere fällt aus dieser Sicht in die Dimension einer illegitimen Statthalterschaft. Angefangen beim Einfall der Römer und dem Konflikt zwischen den Zeloten und dem Römischen Reich (Hengel 1976) - der sich als ursprüngliche Figur eines immensen Konfliktes darstellt - geht es stets um die Frage des legitimen und des fiktiven Archons. Letzterer ist die wahre Figur der Ideologie bzw. die verkörperte Ideologie. Der illegitime Archon repräsentiert den Fürsten ohne Charisma, der folglich sehr schnell dem Untergang geweiht ist. Er ist eine Figur des Scheins - des ästhetischen Scheins -, denn er will das Ewige symbolisieren, während er nichts anderes ist als ein fiktiver und folglich vergänglicher Herrscher. Die Figur der Ideologie besteht also in der Ersetzung des legitimen Herrschers durch den illegitimen Betrüger, das heißt durch den Doppelgänger, der von Herodes bis zu den Drei Musketieren und der eisernen Maske, eine Art Albtraum darstellt. Die Ideologie und ihre Figur entstehen also daraus, dass ein falscher Archon an die Stelle des legitimen Herrschers tritt. Die Ideologie präsentiert sich somit von Anfang an in der Form des scheinbaren Doppelgängers und dies führt uns von Herodes über Hoffmanns Märchen Klein Zaches genannt Zinnober bis zu Hitler in der Satire Chaplins und schließlich zur Erklärung der Bekennenden Kirche Deutschlands, die dem Führer jede Legitimität abspricht.

\section{Die instabile Macht}

Der allgemeine Rahmen zeigt uns eine konstitutiv instabile Macht, die auf einer dynamisch-konfliktreichen Struktur basiert. Die Legitimität der Macht geht in der Fähigkeit auf, sich zu verdoppeln und eine unvergängliche Darstellung ihrer selbst zu schaffen, die über die vergängliche Dimension hinausgeht. Es handelt sich im Grunde um eine ästhetische Legitimation, denn durch einen Widerschein bzw. durch eine Selbstdarstellung wird das Geschehen nachträglich sanktioniert. So bewahrt die Machtstruktur Spuren einer relativen, aber systematischen In- 
stabilität und dynamis, die zum Problem ihrer Legitimation zurückführt. Wir haben es weniger mit einer instabilen Macht als mit einer dynamischen Dimension innerhalb der Machtstruktur zu tun, die sich mit transzendenten Symbolen und zugleich mit Symbolen der Transzendenz verbindet. Bisweilen kann es jedoch zu einer Immanentisierung der Transzendenz kommen. Dies ist zum Beispiel bei Karl dem Großen, dem Schwimmenden Souverän, der Fall, wie Horst Bredekamp aufgezeigt hat. Karl der Große mit seiner imposanten Gestalt, der im Wasser der Aachener Quellen Söhne, Optimaten und selbst die Königswache um sich versammelte, übte eine äußerlich zur Schau gestellte Macht aus, die mit seinem Körper zusammenhing und daher sozusagen konjunkturell und instabil war. In dieser eindrucksvollen, aber grundsätzlich fragilen Form hob jene Macht sich gegenüber den Theokratien des Orients ab (Bredekamp 2014). Doch schon die Machtstruktur des Römischen Imperiums, die für spätere Gestaltungen der politischen Macht ein Vorbild war, hing von der außergewöhnlichen Verbindung der Ämter des Pontifex Maximus und des Prätors ab, in der ein juristisches und ein religiöses Motiv zusammenliefen (Monateri 2017).

Die abendländische Macht ist unstet und ihre Sanktion von Anfang an instabil. Doch dies gereicht ihr nicht zum Nachteil. Es handelt sich um eine dynamische Macht und/oder um eine innere dynamis der Macht, die ihre Struktur im Westen im Gegensatz zur starren Struktur des Reichs des Ostens prägt.

$\mathrm{Zu}$ ihrer Bestätigung bedarf diese instabile, konfliktreiche und dynamische Struktur fester Symbole, die die im Hintergrund lauernde Unstetigkeit zurückhalten - und damit kommen wir zur ästhetischen Hinsicht der Frage. Es kann sich um das Bild des Kaisers, aber auch um das Bild der Haartracht der Kaiserin handeln, die sich auf den Mosaiken des ganzen Reichs zur Schau stellt. Das Katechon, die aufhaltende Macht, präsentiert sich (auch) in einer universellen Symbolik, die eben deshalb universell ist, weil sie die Instabilität, die sie nährt, in sich festhält.

Doch was bedeutet in diesem Zusammenhang ,universell‘? Die Transzendenz des Universellen nimmt hier eine andere Gestalt an als die, an die eine bestimmte religiöse Sprache uns gewöhnt hat. Wir müssen den Blick nicht nach oben richten, sondern auf Augenhöhe halten. Diese Symbole sind nur insofern universell, als sie Blicke, die aus unterschiedlichen Winkeln kommen, in einer einzigen facies in sich einschließen. Mit anderen Worten verändert die Perspektive den Bestand der symbolischen Struktur nicht. Dies ist im Grunde der ursprüngliche Kern des modernen Pluralismus.

Das Katechon deutet auf eine höchst aufschlussreiche formale Dynamik hin, wenn sich erweist, dass die Struktur unserer weltlichen Macht konstitutiv instabil und die Krise bzw. der „Ausnahmezustand“, um Carl Schmitt zu zitieren, andauernd ist. Das Katechon verleiht dem Bösen Gestalt und verhindert, indem es 
mit ihm in Berührung kommt, paradoxerweise, dass es in seiner pathologischen Dimension in Erscheinung tritt. Nur wenn das Böse, das als Entropie und Weg hin zum Tod zu verstehen ist, zutage tritt, ist es tatsächlich das Böse und lässt sich besiegen.

Dieser Sachverhalt ist auch und vor allem mit Blick auf die Gegenwart zu betrachten. Um es sehr verkürzt auszudrücken, ist das Katechon das, was uns heute fehlt. Wir sind die Epoche, die keine aufhaltende Macht mehr kennt und die äußerste Krise der Legitimation und Selbstlegitimation erlebt. Von der Antike über die Moderne und Postmoderne sind wir bis zum Anthropozän gelangt: zur Epoche der Energieverschwendung par excellence. Die Frage ist politischer oder, besser gesagt, ästhetisch-politischer Art. Wir müssen uns fragen, wovon die Instabilität abhängt, ob sie zufällig ist, an eine Jetztzeit gebunden, die sozusagen einen exzentrischen Weg eingeschlagen hat, oder ob sie hingegen mit einem ferneren Schicksal zusammenhängt. Mit der Antwort ändert sich die Diagnose der Instabilität unserer Zeit und unserer Formen der Souveränität von Grund auf.

Wir müssen uns wirklich die Frage nach unserer Herkunft stellen und dies erlaubt uns, die Gegenwart zu betrachten. Es geht darum zu verstehen, ob wir plötzlich in einen Abgrund gestürzt sind - einen Ort, der uns fremd ist und in dem wir uns nicht wiedererkennen - oder ob dieser Augenblick schon seit langer Zeit durch eine konstitutive Instabilität der überkommenen Formen der Souveränität vorbereitet wurde. Anders formuliert lautet die Frage: Ist die Flut der zugleich verwirrenden und identitätsstiftenden Bilder, die uns beherrscht, ist die Gesellschaft des Spektakels nach der Definition von Guy Debord historisch betrachtet ein junges bzw. ganz junges Ereignis oder gehören diese Bilder zu einem Weg, der schon lange Zeit währt?

Meine These ist, dass sich die heutigen symbolischen Formen der Legitimation und Identifizierung bzw. des Identitätsangebots als degenerierte Spätformen des Katechon rekonstruieren lassen, sodass auch die Gegenwart in einer aus der „katechonischen Dialektik“ entspringenden Perspektive gedeutet werden kann. Dergestalt lässt sich in der hypertechnologischen Gegenwart, die von einer immer weniger Sicherheiten bietenden Bilderflut überschwemmt wird, das Ergebnis eines langen Weges erkennen, in dem schon das erste Glied instabil war. Instabil war im Übrigen von Anfang an die Dynamik der imperialen Macht. Cäsar bekleidete das doppelte Amt des Pontifex Maximus und des Prätors. Er vereinte die beiden Gesetzgebungen und die beiden Reiche in seiner Person und diese Verbindung blieb dann ein konstitutiver Bestandteil des Kaisertums. Die universelle Verbindung der beiden Reiche war unter diesem Gesichtspunkt stets instabil und provisorisch. Sobald die beiden Elemente auseinanderfallen, bleibt eine Macht ohne Legitimation, eine potestas ohne auctoritas übrig und es entsteht ein ins Leere laufender Legimationsanspruch, der um Leitbilder kreist, die nicht ein- 
halten können, was sie versprechen. Dies ist der heutige Markt der Symbole, der einem zunehmend verwirrten und unsicheren Ich Bilder seiner selbst vermittelt: vom Luxus über den Glamour bis zu den religiösen Identitäten usw.

Die Frage ist kurz gesagt folgende: Hängt die Legitimitätskrise, das heißt die offenkundige und für alle sichtbare Krise der Selbstdarstellungsformen der heutigen Macht, von einem Erdbeben ab, das uns aus dem und über das Symbolsystem des christlichen Abendlandes hinausführt? Oder ist diese Krise nicht vielmehr im Inneren eben dieses Systems angelegt? Im letztgenannten Fall hätten wir es nicht mit einem traumatischen, sondern mit einem seit Jahrhunderten vorbereiteten Ereignis zu tun. Damit verändern sich Blick und Perspektive. Eine Geschichte des Katechon mit Blick auf ihren „apokalyptischen“ Ausgang zu schreiben, bedeutet in diesem Rahmen eine wesentliche Befragung der Gegenwart.

Das Hervortreten einer immer einflussreicheren Dimension des Ästhetischen in unserer Zeit könnte von einer Krise des Katechon abhängen, das in der Zivilisation des Bildes nicht auf seine Vorrechte verzichtet hat, sondern weiterhin Symbole, Identitäten und Identifikationen schafft, die scheinbar universell, in Wahrheit aber immer singulärer und sogar idiosynkratisch und an den eigenen Körper gebunden sind. In der Welt des Untergangs der Eliten, in der die charismatischen Leader einander unaufhörlich abwechseln, lässt sich vielleicht das letzte Erbe des Katechon gewahren. Abgenutzt und verkommen, stellt es sich nunmehr als eine neurotische Bewegung dar, in der die Nichterfüllung der Legitimationsforderung den Untergang des neuen Leaders und den nächsten Wechsel bedingt.

\section{Literaturverzeichnis}

\section{Primärquellen}

Dante Alighieri (1845): Monarchia [ca. 1316]. In: Dantes prosaischen Schriften mit Ausnahme der Vita Nova, 2. Teil. Hg. v. Karl Ludwig Kannegießer. Leipzig: F.A. Brockhaus, 3-26.

E.T.A. Hoffmann (1994): Klein Zaches genannt Zinnober. Reinbek bei Hamburg: Rowohlt. Tertullian (1889): Quinti Septimii Florentis Tertulliani Apologeticus adversus gentes pro Christianis Edited, with introduction and notes by T. Herbert Bindley. Oxford: Clarendon Press.

Tyconius (2004): Liber regularum. Französische Ausgabe: Le livre des règles. Hg. v. J.-M. Vercruuysse. Paris: Editions du Cerf. 


\section{Sekundärquellen}

Blumenberg, H. (1988): Die Legitimität der Neuzeit. Frankfurt a. M.: Suhrkamp.

Bredekamp, H. (2014): Der schwimmende Souverän: Karl der Große und die Bildpolitik des Körpers. Berlin: Wagenbach.

Cacciari, M. (2018): The Witholding Power. An Essay on Political Theology. Transl. by E. Pucci. Introduction by H. Cagyll. London: Bloomsbury.

Hengel, M. (1976): Die Zeloten: Untersuchungen zur jüdischen Freiheitsbewegung in der Zeit von Herodes I, bis 70 n. Chr. Leiden: Brill.

Jünger, E. (1930): Die totale Mobilmachung. In: Krieg und Krieger. Berlin: Junker und Dünnhaupt, 3-30.

Monateri, P. (2017): Augurio. Impero, legge e stato di eccezione. Milano-Udine: Mimesis.

Schmitt, C. (1950): Der Nomos der Erde. Im Völkerrecht des Jus Publicum Europaeum. Köln: Greven.

Schmitt, C. (2007): Briefwechsel 1971-1978 und weitere Materialien. Frankfurt a. M., Suhrkamp 2007. 
